MATEC Web of Conferences 26,02002 (2015)

DOI: $10.1051 /$ matecconf/ 20152602002

(C) Owned by the authors, published by EDP Sciences, 2015

\title{
Preparation and Characterization of Graphene
}

\author{
Man Xu, Yun Zhu ${ }^{1, a}$, Jia-you Ji*, , Fan Shen, and Shu-lin Wang \\ School of Materials Science and Engineering, Wuhan Institute of Technology, Wuhan 430073, China
}

\begin{abstract}
The homogeneous-dispersed graphene oxide was prepared by the improved Hummers method, which would be reduced to graphene with the reducing agent called hydrazine hydrate. The X-ray diffraction, Infrared spectroscopy, Raman spectra and Transmission electron microscopy were used to analysis the phase, morphology and structure of the graphene and graphene oxide. The results show that the graphene oxide and reduced graphene oxide prepared via this method, whose degree of oxidation and reduction are high; Also obtained a higher well-disperses and less structural imperfection of the graphene that was manufactured in the reducing system that added ammonia reduction
\end{abstract}

\section{Introduction}

Graphene is a kind of two-dimensional cellular crystal tightly packed into by the monolayer of carbon atoms, and the perfect graphene which is formed by $\mathrm{sp} 2$ hybridization presents a stable two-dimensional structure, it only has a hexagonal cells. It's another new form of carbon structure that is different from that of the fullerenes and carbon nanotubes [1]. Of course, it is founded after them. 2004, University of Manchester, professor Geim got the high quality of graphene by the method of tape tearing [2]. Since then, graphene has attracted much more researchers to study for its unique properties. Such an approximately perfect structure, such as the electricity [3], thermology [4], mechanism [5], etc., that make graphene own a bright application prospect in the transparent electrode, solar cells, sensors, supercapacitors, energy storage and catalyst carrier, and other fields. Now, the preparation of high quality graphene and the improvement application of graphene has become a hot area of research, also it is the best way to advocate energy-saving environmental protection and social development. Based on the concept of low-cost and large-tonnage, the improved Hummers method is adopted to prepare graphene, and the change of the phase, structure and morphology is studied under the different control condition.

\section{Experiment}

\section{The preparation of graphite oxide.}

The experiment used the improved Hummers method. Measuring $100 \mathrm{ml}$ of concentrated sulfuric acid in the 250 $\mathrm{ml}$ beaker and stirring with the $0{ }^{\circ} \mathrm{C}$ ice water bath, after of the operation, $1.0 \mathrm{~g}$ sodium nitrate was added in the mixture. When it dissolved adequately and stirred uniformly, $3.0 \mathrm{~g}$ flake graphite would be added in batches. $10.0 \mathrm{~g}$ potassium permanganate should be joined in the mixtures slowly after it was stirring for 30minutes to prevent the explosion due to the high local temperature caused by instantaneous heat release. Continuing to reaction 1hour, the beaker was transferred into the $40{ }^{\circ} \mathrm{C}$ water-bath with constant temperature and kept stirring for 2 hours. During high-temperature stage, the reaction was under the conditions of room temperature and agitation. $140 \mathrm{ml}$ distilled water would be added in the form of droplets to maintain the solution under $100{ }^{\circ} \mathrm{C}$. Finally, the oxidation reaction was finished by the addition of 400 $\mathrm{ml}$ distilled water and $5 \sim 10 \mathrm{ml} \mathrm{30 \%}$ hydrogen peroxide to make the color of solution change into golden yellow. The resultant mixture product was filtered while it still hot and washed with $5 \%$ sulfuric acid and distilled water in less frequent way and dried at $60{ }^{\circ} \mathrm{C}$ under vacuum for 48 hours.

\section{The preparation of graphene.}

$0.1 \mathrm{~g}$ graphite oxide was dissolved in $50 \mathrm{ml}$ distilled water and ultrasonicated with power of $100 \mathrm{~W}$ for 2 hours until the solution turned into brown. So the graphene oxide solution was got. And then the graphene oxide aqueous solution was heated up to $80{ }^{\circ} \mathrm{C}$, at the same time, 2 m185\% hydrazine hydrate was added. The mixture would maintain that temperature and stir for 6 hours. All that remains was to filter, wash and dry under vacuum to get the graphene.

\section{Results and discussion}

\section{X-ray diffraction analysis.}

ahuywh712@163.com; ${ }^{\mathrm{b}}$ Corresponding author: 81253640@qq.com

This is an Open Access article distributed under the terms of the Creative Commons Attribution License 4.0, which permits unrestricted use, distribution, and reproduction in any medium, provided the original work is properly cited. 
As shown in figure 1, it can be seen that the graphite oxide show a very obvious (001) peak at $10.3^{\circ}$ for a typical characteristic peak with a layer spacing of 0.859 $\mathrm{nm}$ significantly larger than that of graphite of $0.335 \mathrm{~nm}$. The phenomenon is attributed to the introduction of a large number of oxygen groups of the graphite oxidation make graphite intercalating space larger. The $(001)$ diffraction peak disappeared after reduction of the graphite oxide, thus opposite is appearing a diffraction peak at around $24.5^{\circ}$ which is similar to graphite that is at $26^{\circ}$, but the diffraction peak of graphene is more widely obviously. For the size of graphite is smaller by the reduction reaction and the degree of deficiency and disorder of graphite will increase via the two stages that is oxidation and reduction.

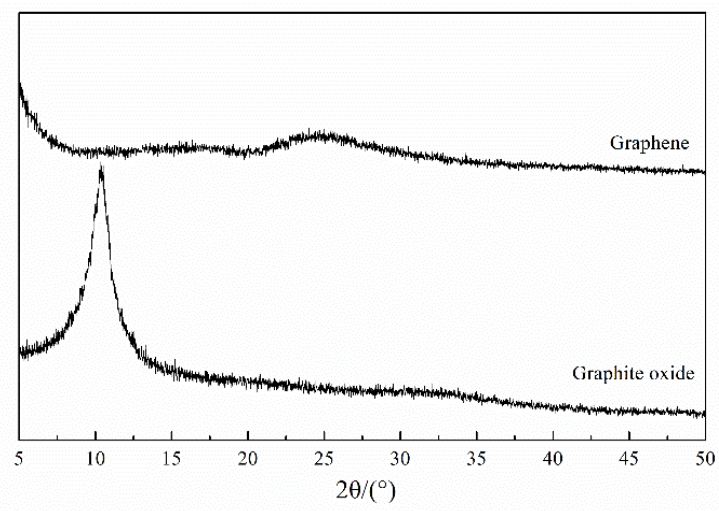

Figure.1. XRD patterns of graphene and graphite oxide

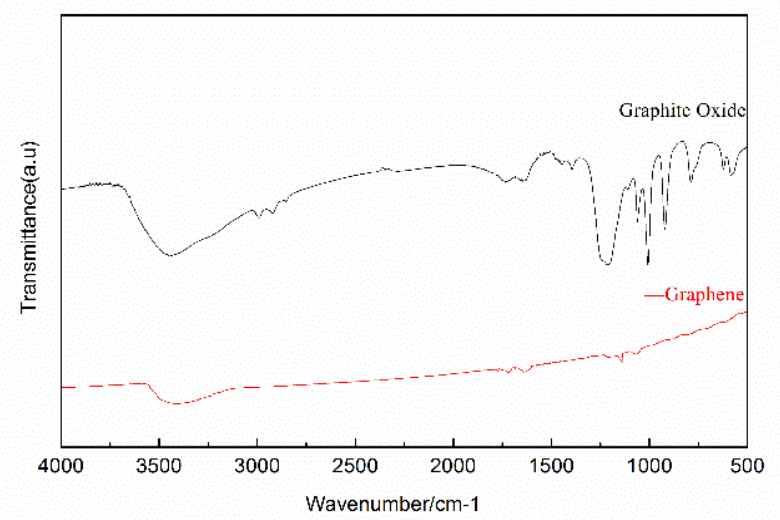

Figure.2. Infrared spectra of graphite oxide and graphene

\section{Infrared spectroscopy analysis.}

The infrared spectra from $500 \sim 4000 \mathrm{~cm}^{-1}$ of the graphene and graphene oxide are shown in figure 2. It is shown that the peak at about $3430 \mathrm{~cm}^{-1}$ attributed to $\mathrm{OH}$ stretching vibration in the high frequency area, and at 3000 3700 $\mathrm{cm}^{-1}$ owing to a wide spectrum peak that mainly because of $\mathrm{OH}$ bending vibration in the water molecules that the graphite oxide absorbs. The peak at about $2900 \mathrm{~cm}^{-1}$ attributed to the $\mathrm{C}-\mathrm{H}$ stretching vibration of $\mathrm{CH}_{2}$ groups, at about $1730 \mathrm{~cm}^{-1}$ belong to the $\mathrm{COOH}$ stretching vibration of carboxyl groups of edge of graphite oxide, at about $1640 \mathrm{~cm}-1$ refer to the $\mathrm{C}=\mathrm{C}$. The peak at the range of $1500 \sim 900 \mathrm{~cm}^{-1}$ might be related to C-O-C stretching vibration on the surface of graphite oxide entirely. Besides the band at $1390 \mathrm{~cm}^{-1}$ ascribed to $\gamma \mathrm{C}-\mathrm{O}-\mathrm{C}$, at $1200 \mathrm{~cm}^{-1}$ belong to $\beta \mathrm{C}-\mathrm{O}-\mathrm{C}$, at $1100 \sim 900 \mathrm{~cm}^{-1}$ and 780 $\mathrm{cm}^{-1}$ are corresponded to $\alpha \mathrm{C}-\mathrm{O}-\mathrm{C}$ and $\mathrm{C}-\mathrm{O}-\mathrm{C}$. These existing oxygen containing groups show that graphite has been oxidation and the degree of oxidation is very high. This is the reason graphite oxide has good hydrophilicity due to the existence of oxygen containing groups. Furthermore, a delamination effect could occur within alkaline solution to apply to the synthesis of composite materials under certain conditions.

Compared with graphite oxide, the absorption peak of oxygen-containing groups vibration of graphene are all disappeared. It is also shown that the reduction degree of graphite oxide is so high.

\section{Raman spectrum analysis.}

The Raman spectrum of graphite, graphite oxide and graphene are shown in figure 3 . The most obvious and important characteristic peak of the single atomic layer graphene is the $\mathrm{G}$ band at about $1580 \mathrm{~cm}^{-1}$ caused by the first-order E2g phonons planar vibration of carbon atoms. The $\mathrm{D}$ band is produced on the edge defects or lattice structure damage in the process of preparation of graphene, otherwise known as defect peak at about 1350 $\mathrm{cm}^{-1}$. It is shown that the $\mathrm{G}$ band of graphite is at 1570 $\mathrm{cm}^{-1}$ and blue shift relative to that of the graphene. This is because the $\mathrm{D}$ band comes from the lattice defects, for the breakage of graphite, it is Raman inactive, namely graphite itself almost does not exist defects. The oxidation make its edge and surface introduce a large number of oxygen-containing groups and change the bond of $\mathrm{C}=\mathrm{C}$, thus the graphite oxide has the Raman active and a strong $\mathrm{D}$ defect peak at $1350 \mathrm{~cm}^{-1}$. At the same time, the destruction of $\mathrm{C}=\mathrm{C}$ and the change of lattice structure make the part of the carbon atoms turn $\mathrm{sp} 2$ hybridization into sp3 hybridization, the result is the attenuation of $\mathrm{sp} 2$ hybridization and the rise of lattice disorder. So that it has a wide $\mathrm{G}$ band at $1600 \mathrm{~cm}-1$ and redshift to the graphite.

Relate to graphene oxide, the graphene blue shift and its $\mathrm{G}$ band, $\mathrm{D}$ band respectively at $1580 \mathrm{~cm}-1$ and $1340 \mathrm{~cm}^{-1}$. The ratio of $\mathrm{I}(\mathrm{D}) / \mathrm{I}(\mathrm{G})$ is larger than that of graphene oxide, it also shows a part of $\mathrm{sp}^{3}$ hybridization have converted to $\mathrm{sp}^{2}$ after the reduction. The figure shows that graphene oxide have been fully reduced basically, it also means the loss of a large amount of oxygen-containing groups make chemical bonds have further changes and the increase of lattice structure defect. 


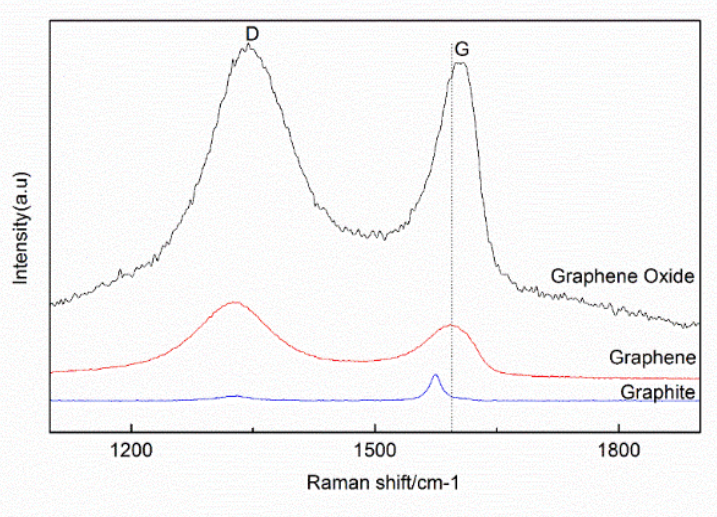

Figure.3. Raman spectrum of graphite, graphene oxide and graphene

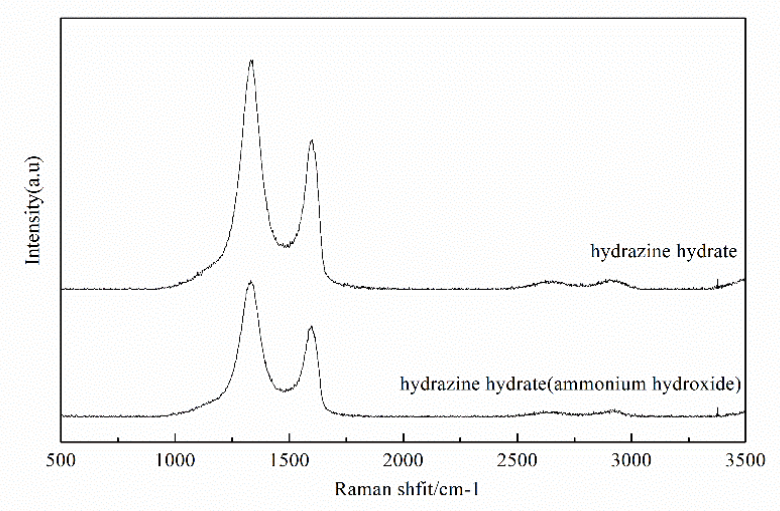

Figure.4. Raman spectrum of graphene with and none ammonium hydroxide

As shown in figure 4, there is no obvious difference in the position of $G$ and $D$ band between the two kinds conditions of preparation of graphene. In the process of preparation, the addition of hydrazine hydrate excessive and alkaline to make the solution present alkaline. But the addition of ammonia did not play an obvious role in adjusting the $\mathrm{PH}$ of solution. On the other hand, compared with both the ratio of $\mathrm{I}(\mathrm{D}) / \mathrm{I}(\mathrm{G})$, we can see the ratio of $\mathrm{I}(\mathrm{D}) / \mathrm{I}(\mathrm{G})$ decrease when add ammonia. It also illustrates the regulation of ammonia can make solution environment become "mild", oxygen containing groups are more likely to fall off, the damage of lattice structure is smaller. All these reflected on the Raman spectrum is the decrease of the ratio of $\mathrm{I}(\mathrm{D}) / \mathrm{I}(\mathrm{G})$. In the experiment, it expresses a better dispersion.

\section{Transmission election microscopy analysis.}

According to information of the picture, the morphology of graphene oxide can be described as flake-like, transparent gauze texture, curly edge and some fold phenomenon. The structure of graphene oxide that is prepared by oxidation - reduction method is hard to avoid some defects that may create incomplete and unsmoothed lamellar structure.

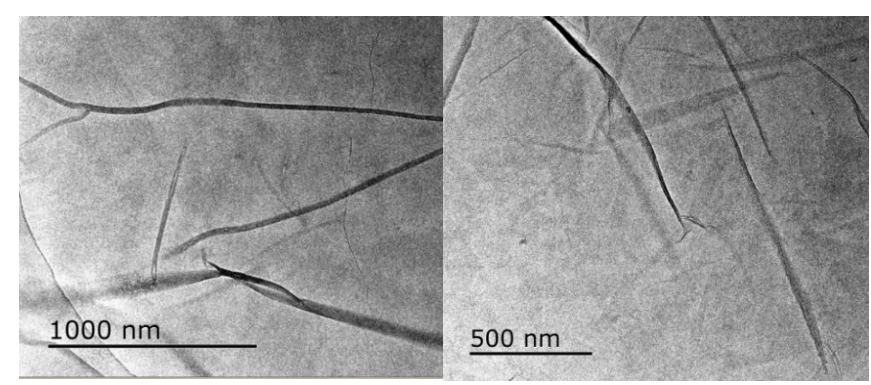

a) Magnification of $1000 \mathrm{~nm}$

b) Magnification of $500 \mathrm{~nm}$
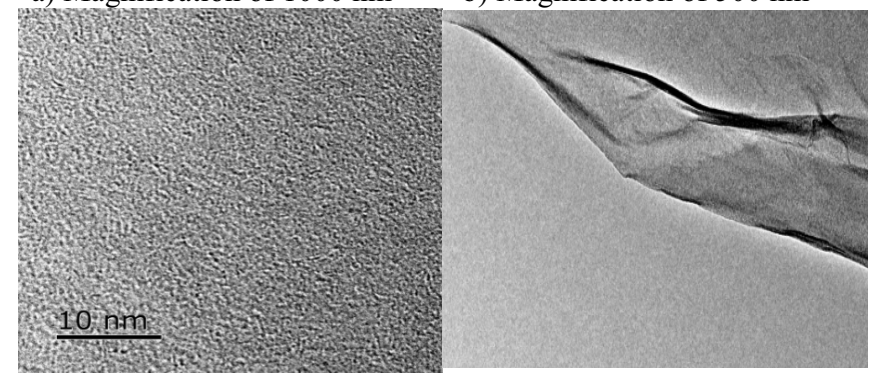

c) Magnification of $10 \mathrm{~nm}$

d)Partial enlarged view of which the magnifying power of $100 \mathrm{~nm}$

Figure.5. TEM picture of graphene oxide

\section{Conclusion}

The degree of oxidation and reduction of the graphene oxide and graphene prepared by improved Hummers method are high and the dispersion of graphene is good. What obtained in this experiment is exactly what we want. Besides, the operation process is not simple, but also it is easy to control.

However, the defects of graphene oxide and graphene are serious and the number of graphene layers more than five. And the reduction mechanism of graphene has not been fully understood. So it requires researchers exert more effort to do further deeper research.

\section{Acknowledgements}

The research was supported by The National Science Foundation of China(NO.21305105), The Science Foundation of Wuhan Institute of Technology(NO.Q201301,201404).

\section{References}

1. Avouris P, Chen Z, Peter beinos V. Nat.Nanotechnol, 2007, 2:605.

2. Sarma S D, Adam S, Hwang E H, et al. Electronic transport in two-dimensional graphene[J]. Reviews of Modern Physics, 2011, 83(2): 407.

3. Kim $\mathrm{T}$ Y, Ruoff R S. High Surface Area Graphene-Based Materials for Electrochemical Energy Storage[C]//Meeting Abstracts. The Electrochemical Society, 2014 (28): 1147-1147. 
4. Balandin A A. Thermal properties of graphene and nanostructured carbon materials[J]. Nature materials, 2011, 10(8): 569-581.

5. Chae H K, Siberio-Perez D Y, Kim J, et al. A route to high surface area porosity and in clusion of large molecules in crystals[J]. Nature, 2004, 427(6974): $523-527$ 